ist, dann muß man auch auf einen Begriff für die Einheit des Faches verzichten.“ (Kieserling, 2004, S. 22 f.)

Ziel der Arbeit ist es nicht, einen einheitlichen Begriff von Soziologie zu entwickeln. Im Gegenteil: Es soll gerade herausgearbeitet werden, wie unterschiedlich das Verhältnis der Soziologie zu ihrem Untersuchungsgegenstand verstanden wird. Dahinter steht die These, dass diese unterschiedlichen Auffassungen Ausdruck einer disziplinären Identitätssuche und eines Selbstverständnisses sind, das unter anderem konstitutiv dafür ist, was unter „guter“ Soziologie verstanden, von der Disziplin als gültiges Wissen anerkannt sowie in interne Debatten aufgenommen wird (Wagner, 1990; Lau, 1984). Denn gerade die Analyse von Grenzdebatten eignet sich letztlich hervorragend, um, im Sinne einer Wissenschaftssoziologie der Soziologie, Einsichten in das Fach selbst zu gewinnen. Denn Grenzen definieren letztlich, was Wissenschaft von Nicht-Wissenschaft unterscheidet:

„Disciplinary boundaries provide the structure needed for a variety of functions, ranging from the allocation of cognitive authority and material resources to the establishment of reliable access to some extra-social reality." (Fuller, 1991, S. 302)

\title{
1.3 DAS DESIDERAT: DIE ANALYSE DER DOPPELSTRUKTUR DER SOZIOLOGIE ALS GRUNDLEGUNG ZU EINER WISSENSCHAFTSSOZIOLOGIE DER SOZIOLOGIE
}

So weit, so gut. Wir sehen uns jedoch in dieser Arbeit mit zwei wesentlichen Herausforderungen konfrontiert, die uns in den weiteren Ausführungen beschäftigen werden. Zunächst ergibt sich die Frage, auf welcher Basis das Grenzproblem der Soziologie untersucht werden kann und mit welchen Mitteln? Das Grenzproblem der Soziologie lässt sich in unterschiedlichster Form beschreiben und zuspitzen. Damit sind nicht nur die diversen wissenssoziologischen, wissenschaftsphilosophischen und -historischen Zugänge gemeint, die unter Punkt 1.2 teilweise bereits angerissen wurden. Es geht auch um die Frage, auf welcher Ebene das Grenzproblem begriffen und dementsprechend auch theoretisch und empirisch gefasst werden kann.

Hier kommen wir zum Kernanliegen dieser Arbeit. Denn stellt man die Frage nach dem Verhältnis der Soziologie zu ihrem Untersuchungsgegenstand neu, so kommt man nicht umhin zu überlegen, auf welcher Basis epistemologischer 
Grundannahmen die Basis für betreffende Aussagen bilden können. Letztlich geht es um die Frage, wie es der Soziologie möglich ist, Aussagen über sich selbst zu treffen. Ein Blick in die Literatur lässt schnell Ernüchterung aufkommen, denn an dieser Debatte zeigt sich exemplarisch, dass die Soziologie über kein adäquates Vokabular verfügt, um ihr Verhältnis zu ihrem Untersuchungsgegenstand selbst zu systematisieren.

Diese Arbeit versteht sich deshalb als Grundlegung zu einer Wissenschaftssoziologie der Soziologie. Obwohl es in der Soziologie zahlreiche Versuche gab, sich selbst zu reflektieren (Wacquant, 1989) und eine Soziologie der Soziologie zu formulieren (Gouldner, 1970; Wagner, 1990; Bourdieu, 1998; Kieserling, 2004), ist bislang kein Programm einer Wissenschaftssoziologie der Soziologie entwickelt worden (Sutter, 2012).

Dabei wäre es durchaus im (professions-)politischen Interesse der Disziplin selbst, mit einem methodisch und theoretisch reflexiven Programm auf Herausforderungen $\mathrm{zu}$ reagieren, die sich der Soziologie seit den letzten Jahren und Jahrzehnten stellen: Impact soll nicht nur von den Natur- sondern auch zunehmend von den Sozialwissenschaften erzeugt werden (Olmos-Peñuela et al., 2014; British Academy, 2008; Bastow et al., 2014; Brewer, 2013; Prewitt et al., 2012; Benneworth et al., 2010). ${ }^{21}$ Politiker seien ,gerade in Zeiten knapper Haushaltsmittel um jeden Hinweis dankbar, der Kürzungsmöglichkeiten legitimiert" (Knoll et al., 2000, S. 22). Nützlichkeitsbekundungen der Sozialwissenschaften sind besonders virulent im Fall von Großbritannien, wo Universitäten im „Research Excellence Framework“ (REF) seit 2014 erstmalig in der Kategorie Impact evaluiert wurden und sich eine nicht unbeachtliche Summe der öffentlichen Finanzierung nach dem Ausgang der Evaluation richtet. Impact umfasst alle möglichen Bereiche wie ,economy, society, culture, public policy and services, health, the environment and quality of life, within the UK and internationally“ (HEFCE, 2014, S. 4):

„It is vitally important that UK policy makers are able to make use of all that humanities and social science research has to offer. By any measure, UK research in the humanities and social sciences is first-class. It generates evidence and findings of high salience for policy makers.“(British Academy, 2008, S. iii)

Wenn auch nicht mit der gleichen Vehemenz wie in Großbritannien, so findet doch auch in Deutschland der Versuch statt, einheitliche Standards für den Wis-

21 Siehe dazu auch die Debatten um die dritte Mission der Universitäten (Etzkowitz et al., 2000) und Mode 2-Wissensproduktion (Gibbons, 1994; Nowotny et al., 2001). 
senstransfer in den Sozialwissenschaften zu entwickeln. Folglich hat der Wissenschaftsrat in diesem Zusammenhang im Jahr 2004 beschlossen, ein Verfahren zum disziplinenspezifischen Rating zu entwickeln, das die „wissenschaftlichen Einrichtungen in Deutschland dabei unterstützen [kann], sich im Wettbewerb besser zu positionieren“ (Wissenschaftsrat, 2005, S. 1). Die Soziologie nahm als eine der ersten Disziplinen am Rating teil, das unter anderem die Kategorien „Wissenstransfer" und „Wissenskommunikation“ erfasst hat. Neidhardt versuchte, auch skeptische Fachkolleg(inn)en für dieses Vorhaben zu begeistern,

„da man davon ausgehen [muss], dass auch unser Fach der sich zunehmend aufdrängenden Zumutung vergleichender Bewertungen gar nicht ausweichen kann. Flächendeckende Evaluationen, in vergleichbaren Ländern schon durchgesetzt und praktiziert, werden auch in Deutschland die Regel werden - ob wir wollen oder nicht. Und wenn das so ist, muss die Soziologie ein professionspolitisches Interesse daran haben, sich gerade den Pilotprojekten solcher Art von Evaluationen nicht zu entziehen“ (Neidhardt, 2006, S. 305).

Obwohl die wissenschaftspolitischen Erwartungen an die Sozialwissenschaften und an die Soziologie gestiegen sind, bleibt sowohl unklar, was in diesem $\mathrm{Zu}$ sammenhang unter Impact (Brewer, 2013; Froese et al., 2016) verstanden werden kann, als auch, was genau mit Innovation gemeint ist (Reinhart, 2016).

Reagiert wird darauf jedoch nicht mit einem reflexiven Forschungsprogramm, sondern mit einer altbekannten Krisenrhetorik (Bonacker et al., 2000), die auf den Bedeutungsverlust der Soziologie hinweist (Knoll et al., 2000; Knie, 2005). Das Fach sei zu stark ausdifferenziert und zu wenig ,interdisziplinierungsfähig“ (Scheffer et al., 2009, S. 291). Die einst vorhandene Reflexivität, die sich in einer Kopplung von Forschungs- und Reflexionsthemen zeigte, ist dem Wachstum und immer neu hinzukommenden speziellen Soziologien gewichen (Kieserling, 2004, S. 18). Das Thema ist gleichbleibend aktuel1 ${ }^{22}$ - trotzdem oder gerade deshalb scheinen jedoch Fachvertreter(innen) davor zurückzuschrecken, ein Programm einer Wissenschaftssoziologie der Soziologie zu entwickeln. ${ }^{23}$

22 Hingewiesen werden kann auf die zahlreichen Debatten in der DGS-Zeitschrift ,Soziologie“, vor allem in ihren Rubriken „Identität und Interdisziplinarität" oder auch „Soziologie in der Öffentlichkeit“. Verhandelt werden unter anderem Themen der „Zukunft der Soziologie“ (Soeffner, 2011), ihre „Interdisziplinierungsfähigkeit“ (Scheffer et al., 2009; Lichtblau, 2010) oder ihre Stellung in der Gesellschaft (Hitzler, 2012; Esser, 2002; Bescherer et al., 2016).

23 Siehe dazu auch die Ausführungen von Kieserling, 2004; Burkart, 2003; Kohli, 1981. 
So begründet sich das Vorhaben dieser Arbeit durchaus wissenschaftspolitisch, jedoch gibt es auch theoretisch-methodologische Gründe für einen solchen Beitrag. Denn eine Wissenschaftssoziologie der Soziologie sollte für die Soziologie aufgrund ihrer Geschichte, ihrer epistemologischen Beschaffenheit und ihres Status einer „sich permanent neu erfindenden Disziplin“ (Sutter, 2012, S. 429) von besonderem Interesse sein (Sutter, 2012).

\subsubsection{Der theoretische Beitrag}

Wie jedoch kann eine solche Wissenschaftssoziologie der Soziologie aussehen? Hier wird argumentiert, dass die Formen der theoretischen Selbstreflexion, die im Fach existieren, nicht ausreichen. So hat es einerseits bereits diverse Unternehmungen gegeben, um eine Soziologie der Soziologie zu entwickeln, die die Begrifflichkeiten des eigenen Fachs zum Gegenstand ihrer Auseinandersetzung machen. Diese lassen sich unterscheiden in historische (Wittrock et al., 1996; Wagner, 1990; Wagner et al., 1994), wissenschaftstheoretische (Bourdieu, 1998) oder wissenssoziologische (Gouldner, 1970; Kieserling, 2004) Fundierungen einer Soziologie der Soziologie. Andererseits ist da die konstruktivistische Wissenschaftsforschung, die Science and Technology Studies (STS), die überwiegend die Naturwissenschaften zum Gegenstand hat und die Sozialwissenschaften erst in geringem Umfang reflektiert hat. Diese Ansätze sind als nebeneinanderher laufend anzusehen und sind bislang keine fruchtbare Allianz miteinander eingegangen.

Grundlegende Voraussetzung, damit eine solche Verbindung fruchtbar sein kann, ist die Annahme einer doppelten Reflexivität des Fachs, die für die Analyse der Soziologie notwendig ist. Die Herausforderung dabei ist, eine Metaperspektive zu konstruieren. Diese ist nicht einfach durch eine Soziologie der Soziologie zu erreichen. Beschreibt sich nämlich die Soziologie mit ihren Mitteln selbst, so besteht die Gefahr, dass epistemologische Vorannahmen und Schlussfolgerungen zirkulär sind. Es ist der Soziologie nicht möglich, aus dieser Perspektive den Standpunkt eines externen Beobachters der Disziplin einzunehmen, da mit der Übernahme eines soziologischen Zugangs auch alle damit verbundenen epistemologischen Annahmen in den Zugang hineinspielen. So gilt es also nicht nur, sich vom eigenen Untersuchungsgegenstand zu distanzieren, sondern auch diverse epistemologische Perspektiven kritisch zu hinterfragen. Denn übernehmen wir die Perspektive von Luhmann oder Giddens in Hinblick auf die Grenzen der Soziologie, so haben wir bereits eine Menge recht essenzialistischer Hintergrundannahmen im Gepäck. Auf der anderen Seite birgt die Fokussierung auf die sozialkonstruktivistische Wissenschaftsforschung im Gewand der STS 
wiederum die Problematik des Relativismus, der eigentlich gar keine Aussagen mehr über die Besonderheit der Soziologie zulässt, da letztlich alles im Fluss und konstruiert ist.

Notwendig wäre deshalb ein Zugang, der die Vorteile beider kombiniert und füreinander fruchtbar macht. Diese stehen jedoch bislang in einem Spannungsverhältnis zueinander. Die Soziologie wirft den STS ein Methodendefizit und eine Theorieaversion vor (Kieserling, 2004, S. 10 f.; Kaldewey, 2013, S. 45). Die Ergebnisse der STS würden sich in zahlreichen, unverbundenen Fallstudien eines eklektischen und interdisziplinär organisierten Forschungsfelds summieren (Kaldewey, 2013, S. 43 ff.). Wissenschaft ist so nichts weiter als Mikropolitik oder Alltagsverhalten. Zudem erlägen die STS einem naiven Empirismus, indem sie sich einerseits auf die eigene Wahrnehmung ihrer Beobachtungen stützen, diese jedoch nicht kritisch hinterfragen. Fotografien oder ähnliches dienten als valide empirische Mittel, um Schlussfolgerungen über epistemische Praktiken abzuleiten (Kieserling, 2004, S. 10 f.). Auf der anderen Seite halten die STS wiederum der Soziologie vor, sie sei essentialistisch und würde weder dem Reflexivitäts- noch dem Symmetriepostulat der STS (Bloor, 1991[1976]) Folge leisten. Zudem besäße sie wenig Erklärungskraft, da sie durch die Trennung von Objekten und Sozialem kaum reliable Aussagen treffen könne (Latour, 2005). Natürlich ist eine solche Kritik mit Blick auf den Entstehungskontext der STS nachzuvollziehen, da diese ja gerade dazu angetreten waren, die Annahmen einer klassischen Wissenschaftssoziologie zu dekonstruieren, welche die Wissenschaft als objektiv und wahr beschrieb (Sismondo, 2010) ${ }^{24}$.

Nimmt man jedoch die Kritik beider Seiten ernst, so geht es zunächst darum, die STS methodologisch zu fundieren und empirische Ergebnisse in einen übergeordneten theoretischen Rahmen einzuordnen. Im Fall der Soziologie hingegen heißt es, ihre Theorien zu epistemologisieren und so neben den Aspekten des Sozialen ihre Grundannahmen im Sinne einer Symmetrie zu reflektieren.

Das Anliegen dieser Arbeit ist, diese beiden bislang unverbundenen theoretischen Stränge zwischen Soziologie und Wissenschaftsforschung anhand zweier Ansätze zusammenzuführen, nämlich der Systemtheorie und dem Ansatz der „boundary work“ (Gieryn, 1983; 1995; 1999), um einen theoretischen Beitrag zu einer Wissenschaftssoziologie der Soziologie zu leisten. Dieser liegt in der kritischen Reflexion und Synthese dieser beiden zunächst unvereinbar erscheinenden Theoriestränge, um deren Vor- und Nachteile für einen solchen Ansatz fruchtbar

24 Siehe dazu auch die Arbeiten von Merton (1973). Eine ähnliche Debatte lässt sich letztlich auch zwischen der Wissenschaftsphilosophie und den STS beobachten. Siehe dazu Reinhart (2012). 
zu machen (Kapitel 2). Grenzen werden in dieser Arbeit gemäß der Auswahl der Theorieansätze als semantische Konstruktionen aufgefasst. Das macht eine Gegenüberstellung wie auch Verbindung dieser Ansätze besonders attraktiv. Im Gegensatz zu einer organisationssoziologischen oder professionssoziologischen Perspektive auf Grenzen (Lamont et al., 2002) ist dadurch die Entwicklung eines wissenschaftssoziologischen Zugangs zur Soziologie selbst möglich. ${ }^{25}$ Der Vorteil dieser Herangehensweise ist zudem, dass weder ein Organisations- noch ein Professions- oder Gesellschaftsbegriff von vornherein zugrunde gelegt werden muss.

\subsubsection{Der methodologische Beitrag}

Hier sollte jedoch eine Wissenschaftssoziologie der Soziologie noch nicht enden. So werden zudem methodologische Implikationen einer Wissenschaftssoziologie der Soziologie reflektiert, die sich in Hinblick auf die Kritik an den STS eben

25 Organisationen wie auch Professionen ziehen Grenzen zu ihrer Umwelt. Organisationen sind in besonderem Maße auf die Akzeptanz ihrer Umwelt angewiesen (Meyer et al., 1977; Hall et al., 1996). Gerade wissenschaftliche Einrichtungen sind zunehmend multiplen Anforderungen ausgesetzt. Universitäten beispielsweise müssen nicht nur ihre Kernaufgaben Forschung und Lehre bewältigen. Ihnen stellen sich zunehmend Aufgaben wie Profilbildung, Qualitätsmanagement sowie Herstellung von Geschlechtergleichheit und Technologietransfer, was auch zu Spannungsfeldern innerhalb der Organisation führen kann (Hasse et al., 2005; Mevissen et al., 2013). Der Begriff Profession entwickelte sich letztlich aus einem Demarkationsproblem, „spezielle“ Berufe von ,gewöhnlichen“ Berufen abzugrenzen (Lamont et al., 2002). Es existieren unterschiedliche Thesen um die Grenzziehungen durch Organisationen und Professionen. Einerseits zeigt sich, dass gerade wissenschaftliche Einrichtungen aufgrund der diversen an sie gestellten Anforderungen sich stärker an ihre Umwelt anpassen, also weniger Grenzen zur Umwelt ziehen können. Inwieweit diese Anpassung jedoch lediglich „window dressing“ (Meier, 2009) ist, wie der Neo-Institutionalismus vermutet, oder ob tatsächlich wissenschaftliche Organisationen zu „normalen Organisationen“ werden, ist bislang umstritten. Meier (Meier, 2009) postuliert, dass auch Universitäten, zumindest diskursiv, immer stärker zu Akteuren werden, wohingegen Musselin (2007) Universitäten nach wie vor als „specific organizations“ beschreibt, die wenig steuerbar sind. Auch die Rolle der akademischen Profession wird zunehmend im Kontext einer Deprofessionalisierung und damit auch von schwindenden Grenzen zur Umwelt diskutiert (Schimank, 2005): Eine Stärkung der Hochschulverwaltungen führt zu einer Schwächung der ,academic oligarchies“ (Schimank, 2005, S. 148). 
nicht in einer Einzelfallstudie erschöpfen. Der methodologische Beitrag dieser Arbeit liegt darin, auf Basis einer hermeneutischen Analyse einen Zugang zu entwickeln, der den Anspruch hat, allgemeingültige Aussagen zu formulieren. Zudem wird die Soziologie als fragmentiertes Feld zum Gegenstand gemacht. Ziel ist es, auf der Grundlage ihrer disziplinären Besonderheiten eine Heuristik zu entwickeln, die ein theoretisches Sampling der Soziologie zulässt (Kapitel 3).

\subsubsection{Der empirische Beitrag}

Der empirische Beitrag dieser Arbeit liegt letztlich in einer kritischen Analyse der Grenzen der Soziologie selbst (Kapitel 4). Das Grenzproblem wird in Hinblick darauf nicht als pure Semantik auf wissenschaftsphilosophischer oder wissenschaftstheoretischer Ebene aufgefasst, wie dies breit angelegte Selbstreflexivitätsunterfangen des Fachs tun (Kieserling, 2004). Es soll vielmehr selbst Teil einer Wissenschaftssoziologie der Soziologie werden. In diesem Sinn findet, im Gegensatz zu theoretischen Analysen, eine Verbindung von „Sache und Person“ (Kohli, 1981, S. 431) statt. Konkret wurden Interviews mit ca. 22 Professorinnen und Professoren sowie Postdoktorand(inn)en der deutschen Soziologie geführt. Die These ist, dass eine solche Verbindung von Sache und Person mehr Aufschluss über das Grenzproblem der Soziologie geben kann als theoretische Analysen. Denn gerade die Trennung von Sache und Person ist eine wirkungsvolle Technik, sich vom eigenen Gegenstand zu distanzieren, also eine Trennung zwischen einem „Innen“ und „Außen“ vorzunehmen. So spricht Burkhart (2003) in diesem Zusammenhang sogar von einem „Thematisierungstabu“ in der Soziologie, der die Subjektivität des Autors wissenschaftlicher Texte von seinem biografischen und sozialen Kontext lösen soll.

Zurück geht der Anspruch der Objektivierung auf Francis Bacon, der die Trennung von Sache und Person als notwendige Voraussetzung für die Wissenschaftsentwicklung sah (Bacon, 2009[1620;1858]; Kohli, 1981; Kitcher, 2001). ${ }^{26}$ Ziel der Trennung von Sache und Person war es, eine wissenschaftliche Aussage allein auf Basis der empirischen und rationalen Methode möglich zu machen (Bacon, 2009[1620;1858]; Kohli, 1981). Wissenschaft sollte nicht mehr auf der persönlichen Autorität Einzelner aufbauen, sondern allein auf der Sache, die nicht - und hier bezieht Kohli sich auf Natorp (1921) - „Meinungssache“, sondern ein „rechtschaffenes Werk“ (Kohli, 1981, S. 431) darstellt.

26 Formuliert hat Bacon diesen Gedanken in seinem Vorwort des Novum Organum: „De nobis ipsis silemus“ (,,von uns selber schweigen wir“) (Bacon, 2009[1620;1858]). 
Dennoch ist in der neueren Wissenschaftsforschung die Verbindung von Sache und Person ein wichtiges Anliegen. Im hier vorliegenden Fall ist damit möglich, das Grenzproblem als offene Situation aufzufassen, die von den Forschenden in Form unterschiedlicher Orientierungen und Befürchtungen ausdefiniert und dementsprechend gestaltet wird (Kohli, 1981). Denn gerade das Ringen um Wahrheit kann Aufschluss über Diskontinuitäten und letztlich die Entstehung bestimmter Narrative um Wahrheit geben. ${ }^{27}$ Der empirische Mehrwert eines solchen Perspektivwechsels liegt darin, dass die vermeintlich glatte Oberfläche eben genannter theoretischer Debatten im Sinne einer Dichotomisierung oder Dialektik diverser Grenzbegriffe einer vielschichtigen Problematisierung weicht, das Grenzproblem also selbst soziologisiert wird. Die Ergebnisse zeigen, dass es sich um wesentlich mehr als theoretische Auseinandersetzungen handelt. Das Grenzproblem hat Einfluss darauf, wie Soziolog(inn)en ihr Fach verstehen und in welchen Konflikten sie sich in Hinblick auf ihr Disziplinenverständnis befinden. Der theoretische Mehrwert einer solchen Perspektive liegt darin, dass sie wiederum Rückschlüsse auf die in der Literatur dargestellte Innen-AußenUnterscheidung des soziologischen Grenzproblems zulässt.

27 Dennoch sind Analysen der Soziologie dieser Art eher rar. Prominentes Beispiel ist Pierre Bourdieus Analyse des soziologischen Feldes in Frankreich, in der er sich unter anderem den Sozialwissenschaften widmet und Charakteristika spezifiziert, die für den Aufstieg im französischen Elitesystem notwendig sind (Bourdieu, 1984). Mit der Entwicklung einer Theorie der Praxis hat Bourdieu zudem seine meisterhafte Theoriearbeit mit der Verbindung von Sache und Person geltend gemacht (Burkart, 2003). Nimmt man den Standpunkt einer solchen Theorie der Praxis ein, so liegt das Erklärungspotenzial einer Verbindung sogenannter Mikro- und Makrostrukturen bzw. des Subjektivismus und Objektivismus auf der Hand. So ist es umso verwunderlicher, dass sich seit dem Erscheinen von Kohlis Aufsatz im Jahr 1981 in Sachen qualitativer empirischer Analyse der Soziologie selbst erstaunlich wenig getan hat. Einige der wenigen Beispiele sind die Arbeiten von Heilbron (2011), der soziokognitive Mechanismen der Theoriekonstruktion am Beispiel Pierre Bourdieus analysiert. Er verbindet also im Sinne Kohlis (1981) Sache und Person und führt Bourdieus Prinzipien der Theoriebildung auf seine Lebensumstände und seinen Lebenslauf zurück, insbesondere auf seine Rolle im intellektuellen Umfeld in Paris und seine Art der Arbeitsorganisation. Zwar ist die Verbindung von Sache und Person in den Science and Technology Studies, die seit den 1980er-Jahren entstanden sind (Knorr-Cetina, 1981; Latour et al., 1986; Traweek, 1988), schon fast ein alter Hut, dennoch ist auch hier eine gewisse $\mathrm{Zu}-$ rückhaltung in der Untersuchung der Sozialwissenschaften und insbesondere der Soziologie selbst zu beobachten. 
Bevor in Punkt 1.4 der Argumentationsgang der Arbeit dargelegt wird, soll zunächst noch auf eine Begriffsklärung im Rahmen der Arbeit eingegangen werden. Es mag den Lesenden bereits aufgefallen sein, dass der Begriff der Sozialwissenschaften oft in einem Atemzug mit der Soziologie verwendet wurde. Hier fällt die Trennung vor allem in Hinblick auf die Literaturlage schwer. Denn die „Konnotation von Sozialwissenschaften als eine Gruppe von Disziplinen birgt [...] analytische Schwierigkeiten“ (Sala, 2012, S. 261). Die akademischen Fächer, die den Sozialwissenschaften zugerechnet werden, stellen keine „stabile Größe“ (Sala, 2012, S. 262) dar, sondern zeichnen sich vielmehr durch ihre „semantische Mutabilität" (Sala, 2012, S. 262) aus. So wird in der Literatur meist eine nicht begründete Auswahl an Disziplinen genannt wie Statistik, Psychologie, Wirtschaftswissenschaften, Politikwissenschaften, Soziologie, Anthropologie und Geografie. Obwohl darüber im Prinzip Einigkeit herrscht, ist diese Größe als analytischer Referenzrahmen nicht bestätigt worden (Sala, 2012, S. 262). Deshalb wird im Folgenden zwar immer wieder auf die Sozialwissenschaften rekurriert, da dies aufgrund der korrekten Darstellung der Literaturlage notwendig ist. Dennoch ist der analytische Referenzrahmen eindeutig die Soziologie.

Dies ist notwendig, da wesentlich mehr Literatur zum Charakter der Sozialwissenschaften verfügbar ist als zu dem der Soziologie. Im Folgenden wird deshalb auf die Literatur verwiesen, die von der Besonderheit der Sozialwissenschaften spricht, da 1. die Soziologie als Sozialwissenschaft anerkannt ist und 2. die Literaturlage zu Soziologie selbst zu dünn ausfällt und ein ausschließliches Rekurrieren auf Literatur allein zur Soziologie auch relevante Teile der Literatur zu den Charakteristika der Soziologie ignorieren würde. Zudem wird auch in der Literatur selbst nicht trennscharf zwischen Sozialwissenschaften und Soziologie unterschieden. Ein gutes Beispiel dafür liefert die bereits in Punkt 1.2.3 zitierte Aussage Giddens': Einerseits spricht er von der „double hermeneutics“ der social sciences, geht im gleichen Absatz jedoch darauf ein, dass „no clear dividing line between informed sociological reflection carried on by lay actors and similar endeavours on the part of specialists" existiert (Giddens, 1984, S. xxxii f., Hervorhebung der Autorin). Worum also geht es Giddens nun genau? Es sei vorab gesagt, dass viele Aussagen dieser Arbeit in der einen oder anderen Form auch auf die Sozialwissenschaften im Allgemeinen zutreffen mögen. Dennoch wird insbesondere im Theorieteil wie auch in der empirischen Betrachtung auf die Soziologie im Speziellen rekurriert und diese als spezifischer Fall für das Grenzproblem konstruiert. Hier sei vor dem unkritischen Übertragen der These auf weitere Disziplinen, die den Sozialwissenschaften zuzurechnen sind, gewarnt. Inwiefern sich die These des Grenzproblems und die weiteren Schlussfol- 
gerungen dieser Arbeit auf die Sozialwissenschaften im Allgemeinen übertragen lassen, muss im Einzelfall geprüft werden.

\subsection{ARGUMENTATIONSGANG DER ARBEIT}

Für eine erste Annäherung wird die Thematik historisch eingeführt. Dies veranschaulicht, dass die Debatten um die Rolle der Soziologie in der Gesellschaft und um ihr Verhältnis zur Gesellschaft keineswegs neu sind. Dazu werden vier Zeitabschnitte betrachtet, in denen jeweils unterschiedliche Grenzziehungsaspekte der Soziologie im Mittelpunkt stehen (2.1). Die Schlussfolgerungen aus diesem Abschnitt zeigen, dass sich um das Verhältnis der Soziologie zu ihrem Untersuchungsgegenstand diverse Semantiken gebildet haben, die sich in Form verschiedener Dichotomisierungen um Grenzziehungen des Verhältnisses der Soziologie zu ihrem Untersuchungsgegenstand gruppieren.

In Kapitel 2.2 wird ein soziologischer Zugang entwickelt. Dabei werden gleichzeitig zwei Perspektivierungen der Untersuchung von Grenzen in der Soziologie geschaffen: Mithilfe der Systemtheorie ist der Blick von „innen“ auf die Soziologie mit dem Vokabular der Soziologie selbst möglich. Im Zentrum steht hier die Verhandlung der Grenzen der Soziologie, die über semantische Sinnerzeugung geschieht. Diese fertigt das System als sogenannte Selbstbeschreibungen mithilfe von Reflexionstheorien an. Dennoch ergeben sich bei der Übernahme dieser Perspektive mindestens zwei Schwierigkeiten. Die erste bezieht sich auf die Selbstreferenz und die ihr inhärente Schwierigkeit, eine Außenperspektive auf die Systemtheorie einzunehmen. Die nächste Schwierigkeit besteht darin, dass die Systemtheorie prinzipiell keine Akteure berücksichtigt.

Die zweite Perspektivierung beschäftigt sich mit dem Blick auf die Soziologie im Sinne einer Außenposition, die auch den Blick auf den Akteur zulässt. Dies erfolgt mit dem sozialkonstruktivistischen Konzept der boundary work (Gieryn, 1983). Im Zentrum stehen sogenannte ,ideologische Selbstbeschreibungen“, die sprachlich die Grenzen zwischen Soziologie und Untersuchungsgegenstand markieren. Obwohl die beiden Perspektivierungen von unterschiedlichen epistemologischen Annahmen ausgehen, finden sich Konvergenzen in der Rolle der Sprache in Hinblick auf Grenzkonstruktionen. Zudem lässt diese fruchtbare Verbindung der Ansätze zu, auf die epistemologischen Herausforderungen und die in 1.3 genannten Gefahren zu reagieren: Sie gewährleistet einen theoretischen Zugang zur Analyse von Grenzen in der Soziologie, der weder zirkulär noch relativistisch ist. 